\title{
The Nyquist Folding Receiver and Zero-Sum Game
}

\author{
Kaili Jiang ${ }^{*}$, Sujuan Chen, and Bin Tang \\ School of Electronic Engineering, University of Electronic Science and Technology of China, Chengdu, Sichuan, P.R. China \\ *Corresponding author
}

\begin{abstract}
-the interaction between radar signals for cooperative targets and the Nyquist Folding Receiver (NYFR) is investigated from a zero-sum (ZS) game theory perspective. Since the subNyquist sampling is a tradeoff in noise folding with signals versus dynamic range of analogy-to-digital converter (ADC), a parameter management optimization model is modeled of them as a ZS game. And simulation results by applying convex optimization algorithm suggest that this model is suitable.
\end{abstract}

Keywords-NYFR; game theory; zero-sum game; sub-Nyquist sampling; dynamic range

\section{INTRODUCTION}

The success of the Nyquist Folding Receiver (NYFR) [1] structure in wideband signals processing have inspired investigation of NYFR, which is an efficient analogy-toinformation (A2I) architecture [2], [3] that folds the broadband RF inputs by subsampling so that can sample with a low-speed ADC. The most significant feature of NYFR is sampling with periodically non-uniform [4] that is the key to folding, by resampling structure with a stream of short radio frequency (RF) pulses and a narrowband ADC. In practice, the degree of nonuniformity and the size of period have a great influence on the output of NYFR. Hence, the selection of parameters is of particularly importance for NYFR.

The application of game theory in parameter optimization of receiver is new, but in radar and communication is not fresh. For example, the interaction between a target and MIMO radar by modeled as a two-person zero-sum game (TPZS) [5]; Reference [6] focuses on the interference and greedily maximizes its individual capacity of various two-user noncooperative games, and so forth. And a zero-sum game is a mathematical representation of a situation in which each participant's gain (or loss) of utility is exactly balanced by the losses (or gains) of the utility of the other participant, by using Venn diagram to show all possible logical relations between a finite collection of different sets. To the best of our knowledge, the game theory or information theory based analysis of the reconnaissance, interference and even electronic warfare (EW) system, however, has not received attention yet, whereas these tend to disclose the nature of the abstract process or choose a proper strategy.

The contributions of this paper are as follows. We suggest the use of a mutual information (MI) [7]-[11] based MI criterion based ZS game to model the aliasing effects from multiple Nyquist zones (NZ) of NYFR and dynamic range of ADC. The variable in NYFR of control to maximize the MI, while the inputs has effects access to minimize the MI. Yet, there are a lot of catching up to do, lest unilateral, hierarchical, and even the Nash equilibria based on game theory in the future.

\section{MODEL}

\section{A. NYFR System}

The NYFR receiving model matrix can be expressed as an underdetermined linear equation

$$
y(t)=\phi x(t)+v(t)
$$

where $\phi \in \square^{K \times N}$ is measurement matrix, and $v(t)$ is complex white white gaussian noise of distribution $N\left(0, \sigma^{2}\right)$. For the purpose of generalizing this discussion, (1) will be turning into digital form which is given by

$$
Y=\mathrm{A} X+V
$$

where $Y=\left[\begin{array}{llll}y[1] & y[2] & \cdots & y[K\end{array}\right]^{T} \in C^{K \times 1}$ is the received

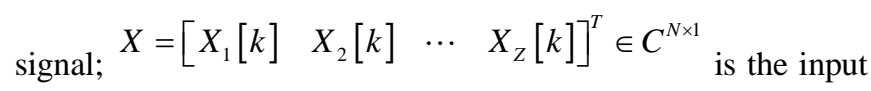
signal $X_{i}[k]=[x[i(K-1)+1] \quad x[i(K-1)+2] \quad \cdots \quad x[i K]]^{T} \in C^{Z \times 1}$ and $i=1,2 \cdots Z$.

Note that $N=K \cdot Z$, where $Z$ denotes index number (namely folding), and $V=\left[\begin{array}{llll}v[1] & v[2] & \cdots & v[K\end{array}\right]^{T} \in C^{K \times 1}$ is the $K \times 1$ receiver noise vector. The measurement matrix $\mathrm{A}$ is shown in [4] as

$$
\mathrm{A}=R S \Psi \Upsilon
$$

$R \in \square^{K \times N}$ is projection matrix, $S \in \square^{N \times N}$ named modulation matrix, $\Upsilon$ and $\Psi$ are the DFT matrix and block matrix of IDFT, respectively.

\section{B. Mutual Information}

Due to the hypothesis that noise is identically and independently distributed gaussian vectors with distribution $V \square N\left(0, \sigma_{v}^{2}\right)$, what' more, the noise vector and output signal vector are also independent of each other, and the covariance matrix of noise is given by

$$
R_{V}=V V^{H}=\left[\begin{array}{cccc}
\sigma_{v}^{2} & 0 & \cdots & 0 \\
& \sigma_{v}^{2} & \cdots & 0 \\
\vdots & \vdots & \ddots & \\
0 & 0 & \cdots & \sigma_{v}^{2}
\end{array}\right]=\sigma_{v}^{2} I
$$


where $I$ is an identity matrix with the size of $K \times K$.

In general, the process of optimization based on NYFR is to find the suitable modulation parameters so that we can detect targets with maximum possible in the interference environment. Therefore, we will take into account the MI which is resulting from $X$ and $Y$ as optimization function of NYFR parameters selection.

The MI of $Y$ relatively to $X$ is defined as

$$
I(X ; Y) \square p(X Y) \log \frac{p(X \mid Y)}{p(X)}=H(X)-H(X \mid Y)
$$

where $p(x)$ is the probability function and, $H(x)$ indicated as entropy of $x$.

From (5) we can see the physical meaning of the MI is that the reducing of uncertainty of input $X$, after obtaining $Y$. In other words, it is the information that $X$ contains in $Y$. As describe in earlier, combining with the measurement matrix A and input signal $X$, we can obtain the information of received signals. But what we all want is recovering signals with high probability, meaning to maximize $I(X ; Y)$, so, the function $\max I(X ; Y)$ is chosen as optimization goal of NYFR.

According to the property of MI and entropy, we have

$$
I(X ; Y)=I(Y ; X)=H(Y)-H(Y \mid X)
$$

where $H(\square)$ is entropy, and measurement matrix $\mathrm{A}$ is the parameter which can be optimized. Expanded $H(Y)$ and $H(Y \mid X)$

$$
\begin{aligned}
H(Y) & =-\int f(Y) \log f(Y) d Y=-E\{\log f(Y)\} \\
H(Y \mid X) & =H(\Phi X+V \mid X)=H(V) \\
& =-\int f(V) \log f(V) d V=-E\{\log f(V)\}
\end{aligned}
$$

where $f(\square)$ denotes the probability density function (pdf).

Note that $V=Y-\Phi X$ obeys a Gaussian $\operatorname{distribution}^{N}\left(0, \sigma^{2}\right)$, so pdf of $Y$ can be written as

$$
\begin{aligned}
f(Y) & =\frac{1}{(2 \pi)^{N} \operatorname{det}\left(R_{V}\right)} \exp \left\{-\frac{1}{2}[Y-\mathrm{A} X]^{H} R_{V}^{-1}[Y-\mathrm{A} X]\right\} \\
& =\frac{1}{(2 \pi)^{N} \sigma_{v}^{2 N}} \exp \left\{-\frac{1}{2 \sigma_{v}^{2 N}}[Y-\mathrm{A} X]^{H}[Y-\mathrm{A} X]\right\}
\end{aligned}
$$

The entropy $H(Y)$ is recast as

$$
\begin{aligned}
& H(Y)=-E\{\log f(Y)\} \\
& =-E\left\{\log \frac{1}{(2 \pi)^{N} \sigma_{v}^{2 N}} \exp \left\{-\frac{1}{2 \sigma_{v}^{2 N}}[Y-\mathrm{A} X]^{H}[Y-\mathrm{A} X]\right\}\right\} \\
& =-E\left\{-\frac{[Y-\mathrm{A} X]^{H}[Y-\mathrm{A} X]}{2 \sigma_{v}^{2 N}}\right\}+E\left\{N \log (2 \pi)+2 N \log \left(\sigma_{v}\right)\right\} \\
& =\frac{1}{2 \sigma_{v}^{2 N}} E\left\{[Y-\mathrm{A} X]^{H}[Y-\mathrm{A} X]\right\}+N \log (2 \pi)+2 N \log \left(\sigma_{v}\right)
\end{aligned}
$$

Since the assumption about noise, the pdf of $V$ is given by

$$
f(v)=\frac{1}{(2 \pi)^{N} \operatorname{det}\left(R_{V}\right)} \exp \left\{-\frac{1}{2} V^{H} R_{V}^{-1} V\right\}
$$

With a similar derivation, we have

$$
\begin{aligned}
H(V) & =-E\{\log f(V)\} \\
& =\frac{\sigma_{v}^{-2(N-1)}}{2}+N \log (2 \pi)+2 N \log \left(\sigma_{v}\right)
\end{aligned}
$$

Combining (6) and (8), the MI of $X$ and $Y$ is

$$
\begin{aligned}
I(X ; Y) & =H(Y)-H(V) \\
& =E\left\{\frac{[Y-\mathrm{A} X]^{H}[Y-\mathrm{A} X]}{2 \sigma_{v}^{2 N}}\right\}-\frac{\sigma_{v}^{-2(N-1)}}{2} \\
& =\frac{1}{2 \sigma_{v}^{2 N}} E\left\{[Y-\mathrm{A} X]^{H}[Y-\mathrm{A} X]-N \sigma_{v}^{2}\right\}
\end{aligned}
$$

We choose sample entropoy to replace MI entropy which is a traditional conception, in order to simplify calculation.

$$
I(X ; Y)=\frac{1}{2 \sigma_{v}^{2 N}}\left\{\frac{1}{N} \sum_{i=1}^{N}\left(y_{i}-\mathrm{A} x_{i}\right)^{H}\left(y_{i}-\mathrm{A} x_{i}\right)-\sigma_{v}^{2}\right\}
$$

For the convenience of discussion, making the objective function into convex function as fellows

$$
f(\xi)=\frac{1}{N} \sum_{i=1}^{N}\left(y_{i}-\Phi x_{i}\right)^{H}\left(y_{i}-\Phi x_{i}\right)-\sigma_{v}^{2}
$$

where $\xi$ is the variable in NYFR contains an average sample rate, index number, modulated function frequency, ADC sample rate and the duration. In terms of detection, the target is finding a most appropriate $\xi$ to maximize $f(\xi)$

$$
\xi=\arg \underset{\xi}{\max } f(\xi)
$$




\section{RESUlTS AND DisCUSSION}

This subsection concentrates on matlab simulation to verify the theoretical analysis. In the first experiment looking at the course of the Nyquist sampling theorem which is a basic rule and points out the least sampling frequency for reconstructing the signals, meeting the need of maximum MI.

We assume that the signal of NYFR is mono-pulse (MP) at $0.65 \mathrm{GHz}$, the amplitude is 1 with zero phase, and the pulse width is $0.1 \mu \mathrm{s}$ us. NYFR architecture can be presented as following in the assumptions setting, the RF sample rate varies about an average rate $f_{s 1}(0.8 \mathrm{GHz} \square 2 \mathrm{GHz})$, while the total index number is 10 and the modulated function is written as $\sin \left(2 \pi f_{\theta} n\right)$ where $f_{\theta}=10 \mathrm{MHz}$. The duration of the signal is given a constant $0.1 \mu \mathrm{s}$ and the ADC samples uniformly at $f_{s 2}$ which is equal to $f_{s 1}$.

As shown in Fig.1, the maximum MI of $Y$ relatively to $X$ occurs at $1.3 \mathrm{GHz}$ that is the Nyquist sampling rate, and the result is $100 \%$ correct based on 800 Monte Carlo simulations. Hence, we have the conclusion that Nyquist sampling rate is the minimum when signals are without aliasing.

The MI of NYFR sampling with different methods includes uniform sampling, periodic non-uniform sampling and random sampling are listed in the table I, note that the MI of random sampling is highest by comparing with others, meaning the uncertainty of $X$ is minimal after receiving $Y$, which requires more complex reconstruction algorithm. In general, the MI of periodic non-uniform sampling and random sampling are similar, but it also depend on the degree of nonuniformity. With the bigger degree of non-uniformity, the MI entropy will be enhanced.

TABLE I. COMPARISON OF MI IN DIFFERENT SAMPLING

\begin{tabular}{|c|c|c|c|}
\hline Ms1 & $\begin{array}{c}\text { Uniform } \\
\text { Sampling }\end{array}$ & $\begin{array}{c}\text { Periodic Non-uniformly } \\
\text { Sampling }\end{array}$ & $\begin{array}{c}\text { Random } \\
\text { Sampling }\end{array}$ \\
\hline $2.0 \mathrm{GHz}$ & 411.65 & 409 & 5888 \\
\hline $1.9 \mathrm{GHz}$ & 402.1 & 398.8 & 2412 \\
\hline $1.8 \mathrm{GHz}$ & 395.79 & 391.5 & 3379 \\
\hline $1.7 \mathrm{GHz}$ & 395.64 & 389.5 & 7280 \\
\hline $1.6 \mathrm{GHz}$ & 409.58 & 399.7 & 408.4 \\
\hline $1.5 \mathrm{GHz}$ & 472.22 & 449.6 & 950.3 \\
\hline $1.4 \mathrm{GHz}$ & 830.91 & 751.9 & 1351 \\
\hline $\mathbf{1 . 3 G H z}$ & $\mathbf{2 e + 8}$ & $\mathbf{2 e}+\mathbf{8}$ & 2361 \\
\hline $1.2 \mathrm{GHz}$ & 611.07 & 602.3 & 896.9 \\
\hline $1.1 \mathrm{GHz}$ & 285.79 & 282.6 & 1859 \\
\hline $1.0 \mathrm{GHz}$ & 214.79 & 213.9 & 1644 \\
\hline $0.9 \mathrm{GHz}$ & 175.11 & 175 & 956.5 \\
\hline $0.8 \mathrm{GHz}$ & 146.2 & 146.3 & \\
\hline
\end{tabular}

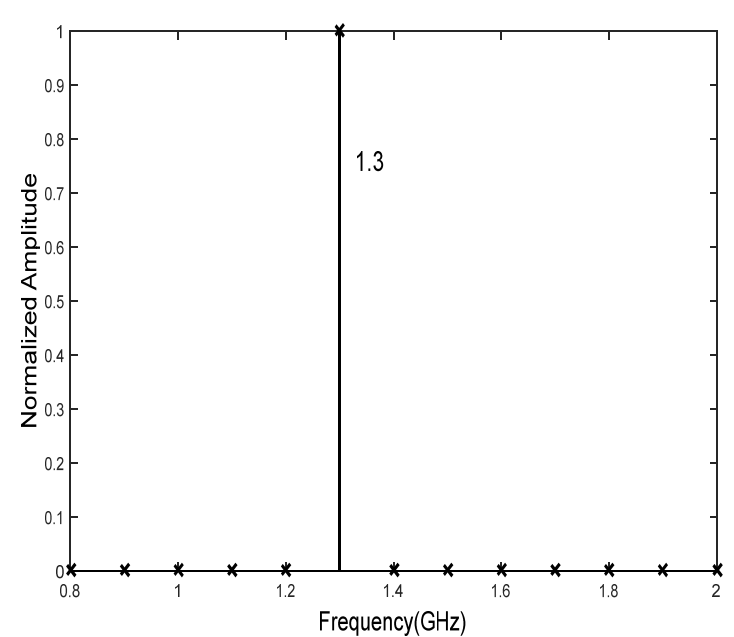

FIGURE I. THE MUTUAL INFORMATION OF RECEIVED SIGNAL RELATIVELY TO INPUT SIGNAL

Another consideration is the parameters' setting of NYFR based on MI optimization guidelines when input is cooperative signals. We performed four experiments to observe the changes of MI where, firstly, for each RF average sampling rate from $0.8 \mathrm{GHz}$ to $2.0 \mathrm{GHz}$ in increments of $0.1 \mathrm{GHz}$, shown in Fig2 (A); secondly in Fig2 (B), for each NZ from 2 to 12 in increments of 1 ; then, Fig.2 (C) illustrates the duration starting at $0.1^{\mu \mathrm{S}}$ and in $0.1^{\mu \mathrm{S}}$ increments $1^{\mu \mathrm{S}}$; and finally in Fig.2 (D), we make the frequency of modulation (with SFM) varies from $1 \mathrm{MHz}$ to $10 \mathrm{MHz}$ which represents the degree of nonuniformity is augmenting. Assume that the signal of NYFR is mono-pulse (MP) at $7.21 \mathrm{GHz}$, other parameters are the same as the first experiment.

As shown Fig.2 (A), with the increasing of the sampling rate, the MI of $Y$ relatively to $X$ is increasing, and at the same time the principle of variation varies with frequency band. But in case of given a fixed average sampling rate, the increasing of index zoom will expand bandwidth of signals can be processed, directly. In Fig 2 (C), the output of NYFR contains more information about the cooperatively inputting signals with the increase of sampling time. Forces on Fig.2 (D), when enlarge the degree of non-uniformity, we can obtain more information about input, by sacrificing the computation and increasing folding noise. As a consequence, the optimization of NYFR system and trace-off are more significance. In addition, multi-parameter joint optimization based on MI can realize the selection of optimal parameters in adaptive system.

\section{FUTURE WORK}

As the model described before, parameters of NYFR optimization based on radar signals for cooperative targets by the ZS game theory perspective. But only a simple model has been included here, we do not consider the adaptive parameters optimization of non-cooperative signals, jamming and the games of unilateral, hierarchical, and even the Nash equilibria solutions. However, as noted, a multi-objective optimization model of NYFR may serve as a better way by regarding each frequency band as an objective function in the future. Certainly, 
according to jamming's respect, we can find out the weak parts of the system.

\section{REFERENCES}

[1] Fudge, R. Bland, M. Chivers, S. Ravindran, J. Haupt, and P.Pace, “A Nyquist folding analog-to-information receiver," in Proc. 42nd Asilomar Conf. on Signals, Compute, Syst. (ACSSC), 2008, pp.541-545E. (references)

[2] Federal Business OpportunitiesD. Healy, "Analog-to-information (A-toI),” DARPA Broad Area Announcement DARPA BAA05-35, Jul. 12, 2005.

[3] Healy and D. J. Bradley, "Compression at the physical interface [The Ato-I and MONTAGE programs],” IEEE Signal Processing. Mag., vol. 25, no. 2, pp. 67-71, Mar. 2008.

[4] J. Wang, S. J. Chen, K. L. Jiang and B. Tang, Frequency Estimation Using SAMP-SVD Based on Nyquist Folding Receiver, has been employed by ICCSP 2015.

[5] T. Basar and G. J. Olsder, "Dynamic Non-cooperative Game Theory", 2nd ed. Philadelphia, PA: SIAM, 1999.

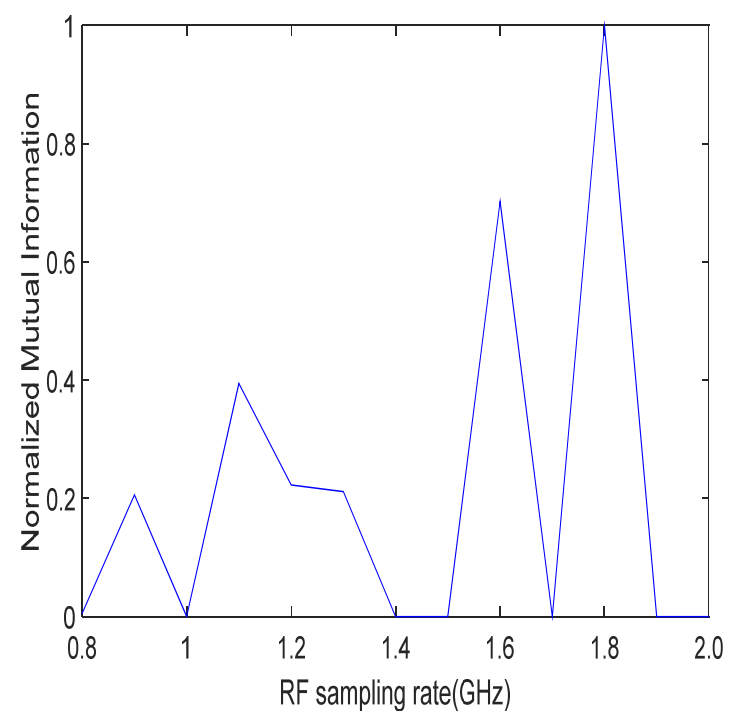

(A)

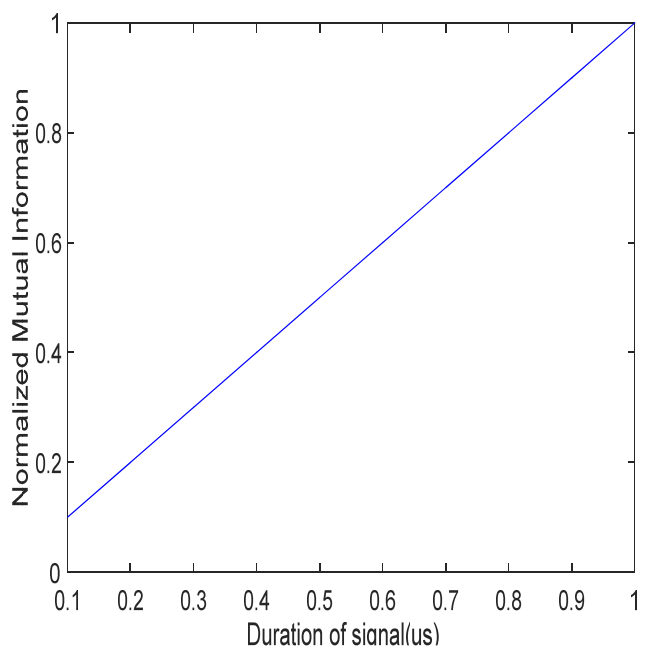

(C)
[6] E. G. Larsson, E. A. Jorswieck, J. Lindblom, R. Mochaourab, "Game Theory and the Flat-Fading Gaussian Interference Channel," IEEE Signal Processing Magazine, 2009 Sep, Vol.26(5), pp.18-27.

[7] Y. Yang and R. S. Blum, "MIMO radar waveform design based on mutual information and minimum mean-square error estimation,” IEEE Trans. Aerosp. Electron. Syst., vol. 43, no.1, pp.330-343, Jan. 2007.

[8] Y. Yang and R. S. Blum, "Minimax robust MIMO radar waveform design,” IEEE J. Sel. Topics Signal Process., vol. 1, no. 1, pp. 147-155, Jun. 2007.

[9] W. Zhang and L. Yang, "Communications-inspired sensing: A case study on waveform design,” IEEE Trans. Signal Process., vol. 58, no. 2 , pp. 792-803, Feb. 2010.

[10] Tang, J. Tang, and Y. Peng, "MIMO radar waveform design in colored noise based on information theory,” IEEE Trans. Signal Process. vol. 58, no. 9, pp. 4684-4697, Sep. 2010.

[11] A. De Maio and M. Lops, "Design principles of MIMO radar detectors," IEEE Trans. Aerosp. Electron. Syst., vol. 43, no. 3, pp. 886-898, Jul. 2007.

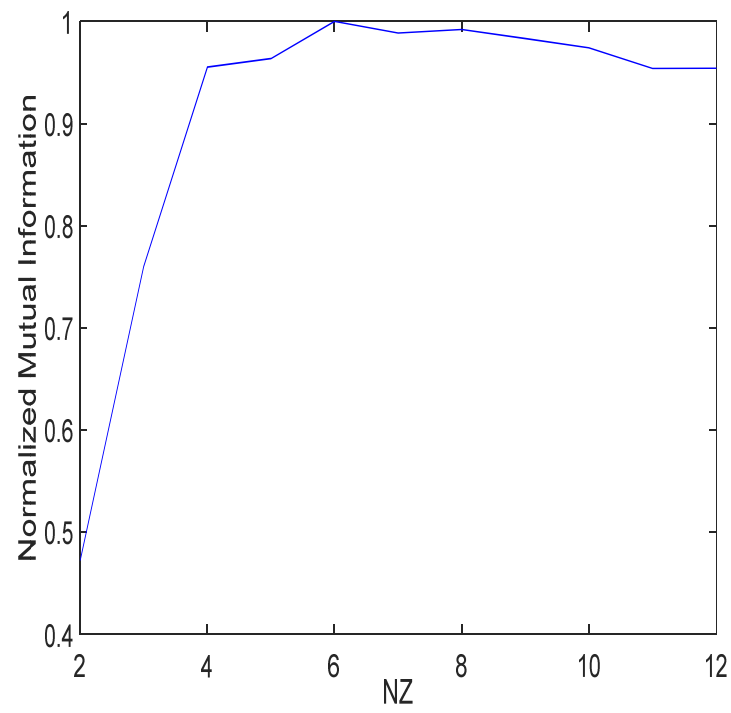

(B)

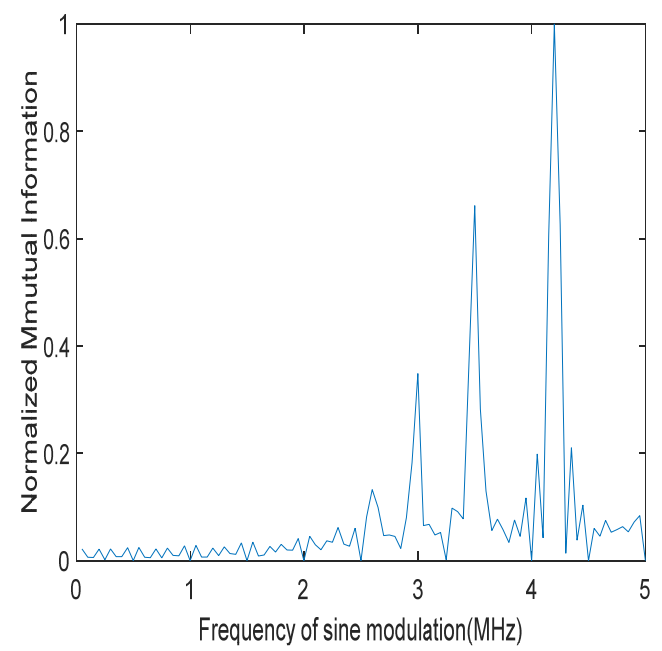

(D)

FIGURE II. THE MUTUAL INFORMATION OF RECEIVED SIGNAL RELATIVELY TO INPUT SIGNAL WITH DIFFERENT PARAMETERS 